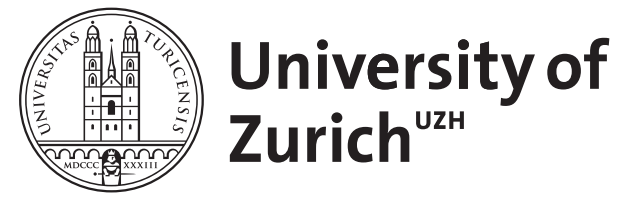

Zurich Open Repository and Archive

University of Zurich

University Library

Strickhofstrasse 39

CH-8057 Zurich

www.zora.uzh.ch

Year: 2010

Rekombinante Allergene. Routinediagnostik oder Wissenschaft?

Schmid-Grendelmeier, P

DOI: https://doi.org/10.1007/s00105-010-1967-y

Posted at the Zurich Open Repository and Archive, University of Zurich

ZORA URL: https://doi.org/10.5167/uzh-43895

Journal Article

Published Version

Originally published at:

Schmid-Grendelmeier, P (2010). Rekombinante Allergene. Routinediagnostik oder Wissenschaft? Der Hautarzt, 61(11):946-953.

DOI: https://doi.org/10.1007/s00105-010-1967-y 
Hautarzt 2010 • 61:946-953

DOI 10.1007/s00105-010-1967-y

Online publiziert: 29. Oktober 2010

(c) Springer-Verlag 2010
P. Schmid-Grendelmeier

Dermatologische Klinik, Universitätsspital, Zürich

\section{Rekombinante Allergene}

\section{Routinediagnostik oder Wissenschaft?}

Anfang der 1990er-Jahre gelang es, aus Allergenquellen einzelne lgEbindende Proteine zu identifizieren $[8,19,21]$. Mittels molekularbiologischer Methoden konnte eine zunehmende Zahl sog. rekombinanter Proteine hergestellt werden. Dadurch wurde es möglich, statt wie bisher eine gesamte Allergenquelle lediglich die IgE-bindenden Proteine und damit Allergene einer solchen Quelle zu verwenden. Diese sog. komponentenbasierte Allergiediagnose kurz CRD nach „,component-resolved diagnosis" - erlaubte, differenzierte Sensibilisierungsmuster und Kreuzreaktivitätsphänomene auf molekularer Stufe zu untersuchen. Solche molekulare Allergene stehen nun auch in zunehmendem Maße zur klinischen Anwendung zur Verfügung. In diesem Beitrag werden der aktuelle Nutzen dieser komponentenbasierten Allergiediagnose [8] in der Praxis und mögliche zukünftige Anwendungen beleuchtet.
Komponentenbasierte Diagnose bei Inhalationsallergien

\section{Pollinose - Majorallergene sind entscheidend}

Heutzutage ist eine Vielzahl an rekombinanten oder natürlich hergestellten molekularen Allergenen aus Pollen bekannt und größtenteils auch kommerziell erhältlich. Hier können für den klinischen Alltag v. a. durch die Abgrenzung von sog. Haupt- oder Majorallergenen zu Nebenallergenen wertvolle Zusatzinformationen gewonnen werden.

Majorallergene sind für die entsprechenden Pollengruppen charakteristisch und in der überwiegenden Anzahl der Fälle auch für die Auslösung der klinischen Beschwerden verantwortlich. Bekannte Majorallergene sind für Birke das Protein Bet v 1 und für Gräserpollen Phl p 1 und Phl p 5. Für Eschenpollen wird als Markerallergen oft das der in hohem Maße kreuzreagierenden Olivenpollen - Ole e 1 - angesehen. Für Beifußpollen gilt Ar v 1 als das wichtigste Allergen [19, 22], das auch in hohem Maße mit einem Hauptallergen von Traubenkraut (Artemisia, Ragweed) Amb a 4 kreuzreagiert [11].

Nebenallergene sind Panallergene, die in einer Vielzahl von Pollen in hohem Maße vorkommen und daher Kreuzreaktionen unter Pollen auslösen. Meist verursachen diese aber deutlich weniger klinische Beschwerden. Sie können daher Hauttest und v. a. auch die In-vitro-Diagnostik stark beeinflussen, sind aber für die Symptome des Patienten nur selten relevant. Solche Neben- oder Panallergene bei Pollen sind insbesondere Profiline und Kalzium-bindende Proteine, kurz Polcalcine genannt. In $\bullet$ Abb. 1 sind die wichtigsten Haupt- und Nebenallergene von Pollen aufgezeigt.

\section{Komponentenbasierte Diagnose als Hilfestellung bei der Indikation zur SIT}

In den zur SIT verwendeten Extrakten sind v. a. die Hauptallergene in gleichbleibender ausreichend hoher Menge vorhanden sind.

\section{- Die SIT ist v. a. bei Vorliegen einer Sensibilisierung auf die Hauptallergene sinnvoll.}

Dies umso mehr als der Gehalt an $\mathrm{Ne}$ benallergenen von Hersteller zu Hersteller stark schwanken kann $[6,7]$. Entscheidend ist daher, ob eine Sensibilisierung auf die Hauptallergene vorliegt. Lässt sich erhöhtes sIgE gegen die GräserpollenMarkerallergene Phl p 1 und Phl p 5 nachweisen, ist dies ein Hinweis auf eine spezifische Sensibilisierung, bei der eine SIT sinnvoll ist. Liegt hingegen ausschließ-
Abb. $1<$ Wichtigste Haupt- und Neben- te Haupt- und Neben-
allergene von Pollentativ fur korbbliter

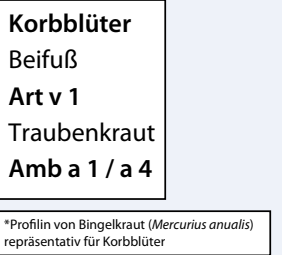


lich erhöhtes sIgE gegen Kreuzallergene wie das Polcalcin Phl p 12 und das Profilin $\mathrm{Phl} \mathrm{p} 7$ vor, ist eine SIT wenig Erfolg versprechend und daher nicht indiziert. Ähnlich ist eine SIT bei Birkenpollen-assoziierter Rhinokonjunktivitis sinnvoll, wenn IgE gegen das Markerallergen Bet $\mathrm{v} 1$ vorliegt, bei einer alleinigen Sensibilisierung gegen die Kreuzallergene Bet $v 4$ und Bet $v 2$ dagegen nicht. Ausschließliche Sensibilisierung auf die Panallergene Profilin/Polcalcine sind eher selten, umfassen aber je nach Kollektiv doch 10-25\% der Pollinosepatienten [8].

In eigenen Untersuchungen berichteten 746 Patienten bei $73 \%$ aller mittels SITbehandelten mit Sensibilisierung auf das Majorallergen über einen guten bis sehr guten Erfolg, aber nur bei 16\%, wenn lediglich eine Sensibilisierung auf Nebenallergene vorlag (• Tab. 1). Die CRD-basierte Diagnostik scheint also tatsächlich Sinn zu machen, um insbesondere für die Pollen-SIT ungeeignete Patienten selektionieren zu können und dadurch neben dem großen Zeitaufwand allfällige Neben- wirkungen und letztendlich auch unnötige Kosten zu vermeiden. Dabei können Sensibilisierungen auf Profilin und Polcalcin durchaus summarisch erfasst werden, da im klinischen Alltag eine Abgrenzung auf eine der beiden Gruppen nicht notwendig ist. So ist die kombinierte Bestimmung etwa von $\mathrm{Phl} \mathrm{p} 7 / \mathrm{Phl} \mathrm{p} 12$ als eine einzelne Bestimmung oft ausreichend. Aufgrund der hohen Kreuzreaktivität werden dadurch auch Sensibilisierungen auf die entsprechenden Panallergene anderer Pollengruppen wie Baumoder Kräuterpollen miterfasst und müssen daher nicht gesondert bestimmt werden (• Abb. 2).

$\mathrm{Zu}$ wissenschaftlichen Zwecken lassen sich durch komponentenbasierte Diagnose auch Rückschlüsse auf unterschiedliche Sensibilisierungsmuster in verschiedenen Bevölkerungsgruppen nachweisen. Grund dafür mögen verschiedene primäre Sensibilisierungswege sein [1]. Zudem können pollenassoziierte Nahrungsmittelallergien etwa zwischen Birkenpollen und rohem Stein- und Kernobst und anderen Nah- rungsmittel in überwiegendem Maße auf Kreuzreaktion zwischen verschiedenen Bet-v-1-Homologen, dem sog. PR („pathogenesis-related protein family“)-10Protein, zurückgeführt werden.

\section{Andere Inhalationsallergien}

Auch bei Milbenallergien lassen unterschiedliche Sensibilisierungsmuster etwa auf Der p 1, Der p 2 oder Der p 10 (Tropomyosin) gewisse Rückschlüsse etwa auf den Sensibilisierungsweg und Kreuzreaktivitäten zu. Bei Tierhaarallergien spricht eine Sensibilisierung gegen Lipocaline oder Serumalbumin für eine breite Reaktionsbereitschaft gegenüber sämtlichen behaarten Tieren, während eine reine Sensibilisierungen etwa gegen Katzen-Uteroglobin Fel d reine ausschließliche Katzenallergie erwarten lässt. Sowohl bei Milben- als auch Tierhaarallergien ist heutzutage der Einsatz von rekombinanten Allergenen aber noch vorwiegend zu wissenschaftlichen Zwecken und weniger in der alltäglichen Praxis einzuordnen.

\section{Hier steht eine Anzeige.}

\section{算 Springer}


Beschwerden im Frühjahr (März /April)

\begin{tabular}{|c|c|c|}
\hline $\begin{array}{l}\text { Hauptallergen: } \\
\text { Bet v1 (t215) }\end{array}$ & \multicolumn{2}{|c|}{$\begin{array}{c}\text { Kreuzreagierende Nebenallergene: } \\
\text { Bet v2, Bet v4 (t221) }\end{array}$} \\
\hline $\begin{array}{l}\text { Komponenten-basierte } \\
\text { Diagnostik }\end{array}$ & \multicolumn{2}{|c|}{$\begin{array}{l}\text { Eignung des Patienten } \\
\text { für eine Birkenpollen-SIT }\end{array}$} \\
\hline $\begin{array}{ll}\text { Bet v1: } & \text { positiv } \\
\text { Bet v2, 4: } & \text { negativ }\end{array}$ & & GUT \\
\hline $\begin{array}{ll}\text { Bet v1: } & \text { positiv } \\
\text { Bet v2, 4: } & \text { positiv }\end{array}$ & & MITTEL \\
\hline $\begin{array}{ll}\text { Bet v1: } & \text { negativ } \\
\text { Bet v2, 4: } & \text { pos/neg }\end{array}$ & & WENIG \\
\hline $\begin{array}{l}\text { Eher keine SIT mit Birk } \\
\text { Testung auf andere Ba } \\
\text { Eschenpollen nOle e } 1\end{array}$ & $\begin{array}{l}\text { renpollen. } \\
\text { aumpollen, z.B. } \\
\text { (t224) empfohlen }\end{array}$ & \\
\hline
\end{tabular}

Beschwerden im Frühsommer (Mai - Juli)

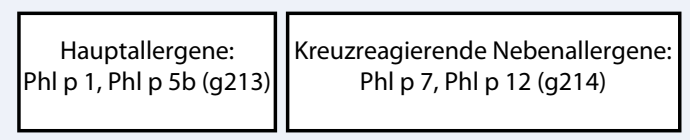

Komponenten-basierte Diagnostik

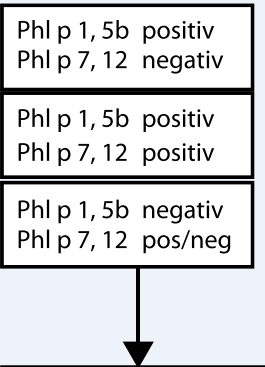

Eher keine SIT mit Gräserpollen

Weitere Untersuchungen, um die sensibilisierende Allergenquelle zu identifizieren
Eignung des Patienten für eine Gräserpollen-SIT

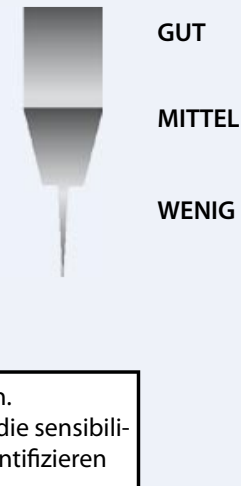

Abb. 24 Komponentenbasierte Diagnostik als Entscheidungshilfe bei der Immuntherapie der Pollinose
Bei der Diagnose von Allergien gegenüber dem Schimmelpilz Aspergillus fumigatus wurde schon 1999 durch Crameri et al. [4] gezeigt, dass krankheitsspezifische Sensibilisierungsmuster bei der allergisch bronchopulmonalen Aspergillose ABPA vorliegen [13]. Bei einer einfachen Aspergillus-Allergie wie bei einer ABPA können zwar IgE gegen Asp f 1 und Asp f 3 nachgewiesen werden; nur bei ABPA finden sich aber Sensibilisierungen gegenüber den Allergenen Asp f 4 und Asp f 6.

\section{Nahrungsmittelallergien - CRD als Hilfsmittel zur Risikoabschätzung}

Zum einen hilft die Bestimmung von IgE gegen Panallergene wie Profiline, aber auch PR-10 Kreuzreaktivitäten besser zu verstehen und genauer zuzuordnen. Auch „Cross-reaktive Carbohydrat-Determinaten" - kurz CCD - finden sich in den allermeisten pflanzlichen Quellen. Eine entsprechende Sensibilisierung auf Bromelin (Ana c 2) oder MUXF3-CCD kann herkömmliche IgE-Bestimmungen verfälschen und ist selten mit klinischen Symptomen assoziiert. Bei einem kleinen Teil der Patienten kann eine CCD-Allergie allerdings schwere Symptome auslösen.
Speicherproteine und Lipidtransferproteine: verantwortlich für schwere anaphylaktische Reaktionen

Im Gegensatz zu den erwähnten PR-10Proteinen und Profilinen gehen Sensibilisierungen auf die meist hitze- und magensäurefesten Speicherproteine oft mit schweren allergischen Symptomen einher. So lassen Sensibilisierungen gegen die Speicherproteine von Erdnüssen (Ara h 1, Ara h 2 und Ara h 3/4), Haselnuss (Cor a 9 und 11) oder gewisse Sojaglycinine ein deutlich erhöhtes Risiko für systemische Reaktionen erwarten als etwa auf die entsprechenden PR-10-Proteine (Ara h 8, Cor a 1 oder Gly $\mathrm{m} 4$ ), die in vielen Fällen nur orale Symptome wie Juckreiz oder Brennen auslösen. Auch Allergien auf Lipidtransferproteine, kurz LTP wie Cor a 8 , Ara h 9 oder das Pfirsich-LTP Pru p 3, gehen oft mit einem erhöhten Risiko für systemische Reaktionen einher.

Letztlich entscheidend ist allerdings auch hier in jedem Fall die Klinik. Auch bei einer „harmlosen“ Sensibilisierung können die Beschwerden den Patienten stark beeinträchtigen - auch wenn diese selten lebensbedrohlich sein dürften. Und andererseits müssen auch Sensibilisierungen auf Speicherproteine oder LPS nicht in jedem Fall zwingend schwere Folgen haben.

Bei Nahrungsmittelintoleranzen, die definitionsgemäß nicht IgE-vermittelt sind, kann naturgemäß keine Diagnose mittels komponentenbasierter Diagnose gestellt werden [18].

\section{Anstrengungsabhängige Beschwerden}

In den letzten Jahren wurde bekannt, dass anstrengungsinduzierte anaphylaktische Beschwerden oft mit einer Sensibilisierung auf das Weizenallergen $\Omega$-5-Gliadin assoziiert sind (Tri a 19, f416; $[3,12]$ ). Bei einem Teil dieser Patienten ist lediglich IgE gegen dieses Weizenallergen Tri a 19, nicht aber gegen Weizen (f 4 ) nachweisbar. Bei typischer "exercice-induced“ Symptomatik kann es daher sinnvoll sein, beide Werte zu bestimmen (engl. „wheatdependant exercice-induced anaphylaxis“, WDEIA). Durch simples Meiden der Kombination von weizenhaltigen Speisen und körperlicher Anstrengung in den nächsten 4-6 h können diese WDEIA-Beschwerden oft vermieden werden.

\section{Kuhmilch, Hühnerei und Meeresfrüchte: tierische Allergene}

Bei der Abklärung einer Kuhmilchallergie kann schon seit vielen Jahren eine komponentenbasierte Diagnostik erfolgen. Gerade bei Caseinsensibilisierung (Bos $\mathrm{d} 8$ ) sind allergische Reaktionen nicht nur auf Milch verschiedenster Quellen wie Kuh, Schaf oder Ziege zu erwarten, sondern u. U. auch beim Genuss des entsprechenden Tierfleisches. 
Im pädiatrischen Bereich sind v. a. bei der Hühnereiallergie durch komponentenbasierte Diagnose weitere Informationen möglich. So zeigen Kinder mit Hühnereiweißallergie mit Sensibilisierung auf Ovomucoid (Gal d 1) oft eine Persistenz dieser Allergie auch über das Kleinkinderalter hinaus. Zudem kann es hier oft auch zu heiklen Reaktionen mit gekochtem Ei kommen. Bei fehlenden oder niedrigen Titern von IgE gegen Ovomucoid sind hingegen oft nur Symptome bei Genuss von rohem Ei zu erwarten [2].

Schließlich sind Tropomyosine für Kreuzreaktionen zwischen Milben (Der p 10) und Meeresfrüchten verantwortlich. Solche Tropomyosine, etwa in Krevetten (Pen a 1, Pen i 1), aber auch in rohem Fisch vorkommenden Parasiten Anisakis (Ani s 3) oder der roten Mückenlarve, können starke Beschwerden bei entsprechendem Genuss oder inhalativer Exposition auslösen.

\section{Latexallergie: Aufschlüsselung auf genuine Allergene}

Eine Latexallergie kann eine Proteinkontakturtikaria bis hin zu schweren, ja lebensgefährlichen allergischen Reaktionen auslösen. Gerade deshalb ist ein positiver IgE-Nachweis auf Latex oft etwas beunruhigend: Soll man dem Patienten für die Zukunft von jeglichem Latexkontakt abraten und einen entsprechenden Allergiepass ausstellen - oder darf er bei entsprechend stummer Anamnese weiterhin mit Latex etwa bei operativen Eingriffen in Kontakt kommen? Hier können durch Bestimmung von einzelnen Latexallergenen sehr hilfreiche weitere Information gewonnen werden kann. Liegt eine ausschließliche Sensibilisierung auf das in Latex enthaltene Profilin (Hev b 8) vor, sind klinisch relevante allergische Symptome auch hier kaum zu erwarten. Solche Sensibilisierungen auf Latex machen bis $\mathrm{zu} 75 \%$ aller positiven Latex-CAPs aus [5, 17]. Hingegen sind $\operatorname{IgE}$ gegen andere genuine Latexallergene ( $\mathrm{rHev} \mathrm{b}_{1}, \mathrm{rHev}_{3}$, rHev b 5 und v. a. auch Hev b 6) in hohem Maße mit eigentlichen allergischen Symptomen assoziiert. Kreuzreaktionen mit dem Allergen Hevein Hev b 6 sind auch für das gelegentlich beobachtete Latex-Frucht-Syndrom verantwortlich.

\section{Hautarzt 2010 • 61:946-953 DOI 10.1007/s00105-010-1967-y}

C) Springer-Verlag 2010

\section{P. Schmid-Grendelmeier \\ Rekombinante Allergene. Routinediagnostik oder Wissenschaft?}

\section{Zusammenfassung}

Die Verwendung rekombinanter Allergenkomponenten eröffnet mehrere diagnostische Möglichkeiten. So können krankheitsspezifische Sensibilisierungsmuster wie etwa bei der allergisch bronchopulmonalen Aspergillose ABPA identifiziert werden. Durch Bestimmung der Majorallergene wichtiger Pollen (Bet v 1, Ole 1, Phl p 1/Phl p 5) kann eine präzisere Indikationsstellung im Hinblick auf eine allergenspezifische Immuntherapie ermöglicht werden, da Extrakte v. a. Majorallergene enthalten. Sensibilisierungen auf Nebenallergene wie Profiline und Polcalcine beeinflussen aufgrund der großen Kreuzreaktivität herkömmliche IgE-Tests, sind aber oft von untergeordneter klinischer Bedeutung. Bei Nahrungsmitteln können häufige Kreuzreaktionen etwa mit Birkenpollen über Bet v 1/PR-10-Proteine nachgewiesen wer- den. Zudem lassen Sensibilisierungen auf Speicherproteine etwa von Erdnuss (Ara h 2) oder Lipidtransferproteine von Pfirsich (Pru p 3) oder Haselnuss (Cor a 8) Rückschlüsse auf ein höheres Anaphylaxierisiko zu. Anstrengungsinduzierte Beschwerden (Tri a 19), unklare Latexsensibilisierungen oder Doppelpositivität bei Insektengiftallergien sind weitere aktuell sinnvolle Einsatzgebiete. Microarray-basierte Allergenchips erlauben bereits heute die Bestimmung von IgE gegen über 100 Allergenen aus kleinsten Serummengen, bedürfen aber noch der Evaluation und Optimierung bezüglich Allergenauswahl und Sensitivität.

Schlüsselwörter

Allergene - Komponentenbasierte Diagnostik (CRD) - Molekulare Diagnose - Microarray . Rekombinante Proteine

\section{Recombinant allergens. For routine use or still only science?}

\section{Abstract}

Component-resolved diagnosis of allergies allows disease-specific patterns of sensitization in some conditions such as allergic bronchopulmonary aspergillosis ABPA). By determination of IgE against important pollen allergens such as Bet v 1, Ole e 1 or Phl p1/Phl p 5 , more precise guidance for allergen-specific immunotherapy may be achieved, as pollen extracts contain mostly these major allergens. Sensitizations against minor allergens such as profilins or polcalcins influence the outcome of IgE measurements against full allergen sources, but are often of limited clinical relevance. In food allergy, frequent cross reactivity between pollens such as birch pollen via Bet v 1/PR10 proteins can be identified. Sensitization against some storage pro- teins such as peanut (Ara $h$ 2) or lipid transfer proteins of peach (Pru p 3) or hazelnut (Cor a 8) may indicate an increased risk of severe anaphylactic reactions. Exercise-induced anaphylaxis, unclear sensitizations against latex or double-positivity in insect allergy are other useful indications for component-resolved diagnosis. Microarray-based allergen chip diagnosis makes possible today the detection of IgE against more than 100 allergens in tiny amounts of serum and is very promising, but still needs evaluation and optimization in regard to allergen selection and sensitivity.

\section{Keywords}

Allergens - Component-resolved diagnosis (CRD) - Microarray - Molecular diagnosis . Recombinant proteins 
Tab. 1 Retrospektive Beurteilung des Erfolgs der SIT und Sensibilisierungsspektrum auf Major- und Minorallergene (anhand von 746 Patienten)

$\begin{array}{llllll}\text { Besserung } & \begin{array}{l}\text { Major }+ \\ \text { Minor }+\end{array} & \begin{array}{l}\text { Major }+ \\ \text { Minor }-\end{array} & \begin{array}{l}\text { Major }- \\ \text { Minor }+\end{array} & \begin{array}{l}\text { Major }- \\ \text { Minor - }\end{array} & \text { Total } \\ \text { Keine } & 24 & 13 & 41 & 6 & 84 \\ \text { Mäßig } & 109 & 28 & 26 & 3 & 166 \\ \text { Gut } & 123 & 137 & 9 & 0 & 269 \\ \text { Sehr gut } & 74 & 147 & 6 & 0 & 227 \\ \text { Total } & 330 & 325 & 82 & 9 & 746\end{array}$

Bestimmte Majorallergene: Bet v 1 und/oder Phl p 1/Phl p 5. Bestimmte Minorallergene: Bet v 2/Bet v 4 oder Phl p 7/Phl p 12.

\section{Insektengiftallergie: CRD nützlich bei Doppelsensibilisierungen}

An anderer Stelle in diesem Heft wird gesondert auf diese Aspekte eingegangen (Beitrag Pryzibilla). Daher soll hier lediglich vermerkt werden, dass durch die Bestimmung von bienenspezifischen (Api $\mathrm{m}_{1}$ ) und wespenspezifischen (Ves v 5) Allergenen eigentliche Doppelsensibilisierungen von lediglich kreuzreagierenden Proteinen oder CCD-Sensibilisierungen abgegrenzt werden können [14]. Das ist besonders bei schweren allergischen Reaktionen von Bedeutung, wenn der Patient das stechende Insekt nicht bemerkt oder identifizieren konnte und der Entscheid zur SIT mit nur einem oder beiden Giften gefällt werden sollte. Weitere allergene Proteine zur Differenzierung der Hymenopterengiftallergie sind bereits in der Entwicklung und werden in naher Zukunft wohl auch kommerziell zur Verfügung stehen.

\section{Hypersensitivität auf Medikamente}

Unverträglichkeiten auf Medikamente sind häufig, sind aber nach wie vor eine diagnostische Herausforderung bei der allergologischen Abklärung. IgE-vermittelte Mechanismen spielen wohl nur bei einer Minderheit eine Rolle. Die entsprechende Bestimmung von spezifischem IgE ist daher selten ausreichend und bedarf weiterer Verfahren wie ergänzenden Hauttests, zellulären Untersuchungen und allenfalls auch Provokationstests. Entsprechend finden sich aktuell noch kaum Untersuchungen mit Allergeneinzelkomponenten.

\section{„Multi-Screening“" mit Einzelallergenen: Diagnose mit Allergenchip}

\section{Wie funktioniert ein Microarray- basierter Allergenchip?}

Mittels kommerziellen Tests lassen sich heute spezifische IgE gegen eine rasch wachsende Zahl rekombinanter oder auch hochgereinigter nativer Allergene bestimmen (z. B. im Phadia CAP oder im Immulite-Verfahren). Mit diesen gängigen Methoden ist es jedoch schwierig, jeden Patienten auf eine große Anzahl unterschiedliche Allergene mit zahlreichen einzelnen ImmunoCAPs zu testen. Dazu ist v. a. der finanzielle Aufwand zu groß. Mittels moderner Biochiptechnologie - sog. Chipanalysen auf Microarray-Basis - können Patienten jedoch auf eine große Zahl von Allergenen simultan getestet werden. Solche Allergenchipmethoden werden die Allergiediagnostik in den nächsten Jahren verändern und neue Wege eröffnen [9].

\section{(7) Mittels Biochiptechnologie können Patienten auf eine große Zahl von Allergenen simultan getestet werden}

Allergen-Microarrays (z. B. der Immuno Solid Phase Allergen Chip, kurz ISAC) bestehen aus einer mit aminreaktiven Polymeren beschichteten Glasoberfläche, die in hoher Dichte Triplikate von bis zu 150 Allergenkomponenten tragen kann. Mittels Nanotechnologie werden dazu geringste Mengen von Einzelallergenen $(<1 \mathrm{ng})$ an einen speziell beschichteten Biochip kovalent gebunden. Während einer 2-stündigen Inkubationszeit mit nur $20 \mu$ Patientenserum binden spezifische
IgE-Antikörper an die korrespondierende Allergenkomponente auf dem Allergenchip und werden mithilfe eines fluoreszenzmarkierten Zweitantikörpers detektiert. Das so entstehende Reaktionsmuster wird mittels eines Laserscanners erfasst und computergestützt ausgewertet, wobei die Fluoreszenzintensität mit der Konzentration allergenspezifischer IgE-Antikörper korreliert. Solche semiquantitative Technologie ist bereits an einzelnen allergologischen Zentren im klinischen (Routine-)Einsatz. Die Resultate werden mittels Computersoftware summarisch zusammengefasst und mit entsprechenden Interpretationshilfen aufbereitet.

\section{Möglichkeiten und Grenzen der Chipdiagnose}

Der Vorteil dieses Systems besteht darin, dass es mittels eines standardisierten Allergenpanels bei jedem Patienten Auskunft hinsichtlich aller relevanten Markerallergene, kreuzreaktiven Allergene sowie seltenen, aber wegen ihres Anaphylaxiepotenzials wichtigen Allergene (z. B. LTPs, Speicherproteine oder Tropomyosin) gibt. Zumindest teilweise kann auch die CCDProblematik eliminiert respektive besser determiniert werden. Ein weiterer Vorteil besteht in der sehr kleinen notwendigen Menge von nur $20 \mu$ Serum, die etwa im pädiatrischen Bereich wertvoll sein kann respektive Bestimmungen mittels Kapillarblutentnahme erlaubt [15].

Auch bei der Erfassung von bestimmten Sensibilisierungsmustern bei der atopischen Dermatitis können solche Chips bestimmte spezifische Muster aufzeigen, die beispielsweise erwartungsgemäß zwischen Kollektiven in Europa und Afrika südlich der Sahara deutlich variieren [[16], Ferrara (Hanifin-Rajka-Meeting 2010)].

Allerdings ist aktuell mittels Chip die ausgezeichnete Sensitivität und Reproduzierbarkeit des CAP-Verfahrens zumindest noch nicht für alle enthaltenen Einzelallergene erreicht. Hier sind sicher noch weitere, auch an großen Kollektiven durchgeführte Untersuchungen zur exakteren Beurteilbarkeit sinnvoll. Auch ist das Allergenspektrum zurzeit noch nicht in allen Bereichen - etwa bei Schimmelpilzallergien - ausreichend und wird daher noch in regelmäßigen Abständen optimiert. 
Selbstverständlich ist auch bei dieser Methode eine profunde Anamnese zwingend und ggf. eine orale Provokation für eine zuverlässige Diagnose in vielen Fällen weiterhin sinnvoll und notwendig.

Für eine sachkundige Interpretation ist zudem aufgrund der Komplexität erhebliche Sachkenntnis notwendig ist. Der breite Einsatz des Allergenchips als primäres Screeninginstrument durch nicht allergologisch ausgebildete Fachärzte ist daher wenig sinnvoll. [9] Ansonsten besteht die Gefahr der Generierung einer Vielzahl an positiven Testbefunden bei gänzlich fehlender Anamnese, was zu Verwirrung und möglicherweise unnötigen Zusatzuntersuchungen führen kann.

\section{(? Die komponentenbasierte \\ Diagnostik ergänzt einen} Hauttest in bester Weise

In unserer Allergiestation untersuchen wir den Einsatz dieses Chipverfahrens seit 2007 an bisher etwas über 300 Patienten. Dabei zeigen sich die Vorteile eines solchen breiten Approaches etwa bei unklaren Anaphylaxiefällen, bei Latexsensibilisierung und bei sehr breiter Sensibilisierung, die klinisch und serologisch möglicherweise auf Panallergene zurückzuführen sind. So lassen sich Sensibilisierungen etwa auf Gruppen von Profilinen, Tropomyosinen oder LTP oft sehr klar identifizieren. Bei Patienten mit Beschwerden bei Nahrungsmitteln mit sehr breiten oder unklaren Auslösern kann eine Chipuntersuchung helfen, solche relevanten Allergengruppen zu identifizieren oder eine IgE-vermittelte Sensibilisierung mit hoher Wahrscheinlichkeit auszuschließen. Auch zeigt sich in präliminären Studien, dass sich möglicherweise die Chipdiagnostik für die Verlaufsbeobachtung während der allergenspezifischen SIT einsetzen lässt, weil die IgE/ IgG4-Ratio in deutlich höherem Maße als bei der einfachen IgE-Bestimmung mittels CAP erfasst wird (Gay-Croiser). Unserer Meinung ersetzt diese oder jegliche komponentenbasierte Diagnostik weder eine sorgfältige Anamnese noch den Hauttest, sondern ergänzt diesen in bester Weise. Auch muss festgehalten werden, dass aktuell mittels Chipuntersuchungen trotz der großen Fortschritten in den letzten 


\begin{tabular}{|c|c|c|c|}
\hline Allergie auf/im & Allergenquelle & $\begin{array}{l}\text { Sinnvolle Kompo- } \\
\text { nenten }\end{array}$ & $\begin{array}{l}\text { Nützliche lgE-Testkombina- } \\
\text { tionen }\end{array}$ \\
\hline Frühjahr & Birke/Esche & Bet v 1/Ole e 1 & Bet $v 1$ und Ole e 1 \\
\hline Frühsommer & Gräser & Phl p 1/p 5 & Phl p 1/p 5 \\
\hline Spätsommer & $\begin{array}{l}\text { Beifuß (Trau- } \\
\text { benkraut) }\end{array}$ & Art $v 1 /$ Amb a 1 & $\begin{array}{l}\text { Beifuß, falls positiv und Sensi- } \\
\text { bilisierungsweg unklar: } \\
\text { 2. Art v } 1 / \text { Amb a } 1\end{array}$ \\
\hline \multirow{2}{*}{$\begin{array}{l}\text { Birkenpollen-assozi- } \\
\text { ierte Nahrungsmittel- } \\
\text { allergie }\end{array}$} & $\begin{array}{l}\text { Rohes Stein- } \\
\text { und Kernobst }\end{array}$ & $\begin{array}{l}\text { Pathogenesis-related } \\
\text { Proteinfamilie } 10\end{array}$ & \multirow[t]{2}{*}{$\begin{array}{l}\text { Bet v 1, evtl. speziesspezi- } \\
\text { fisches PR-10-Protein }\end{array}$} \\
\hline & Nüsse, Sellerie & PR-10-Proteine & \\
\hline $\begin{array}{l}\text { Breite Sensibilisierung } \\
\text { Bei meist geringer } \\
\text { Klinik }\end{array}$ & Pollen, Früchte & $\begin{array}{l}\text { Profiline, Polcalcine } \\
\text { Crossreagierende } \\
\text { Carbohydratgr. }\end{array}$ & $\begin{array}{l}\text { Z. B. Bet v } 2 / v 4 \\
C C D\end{array}$ \\
\hline ABPA & A. fumigatus & Asp $\mathrm{f} 4 / \mathrm{f} 6$ & $\begin{array}{l}\text { A. fumigatus, falls positiv: } \\
\text { 2. Asp } \mathrm{f} 4 / \mathrm{f} 6\end{array}$ \\
\hline Hühnerei & Hühnerei & Ei, Ovomucoid & $\mathrm{Ei}, \mathrm{Gal} d \mathrm{~d}$ \\
\hline Kuhmilch & & Kuhmilch, Casein & $\begin{array}{l}\text { Kuhmilch, falls positiv: } \\
\text { 2. Casein }\end{array}$ \\
\hline $\begin{array}{l}\text { Anstrengungsindu- } \\
\text { zierte Beschwerden }\end{array}$ & Weizen & $\Omega-5$-Gliadin & Weizen, Tri a 19 \\
\hline \multirow[t]{4}{*}{ Anaphylaxie nach } & Pfirsich & Prup 3 & Pfirsich, Pru p 3 \\
\hline & Haselnuss & Cor a 8 & Haselnuss, Cor a 8 \\
\hline & Erdnuss & $\begin{array}{l}\text { Ara h 2, auch Ara } \\
\text { h } 1 / \text { h } 3\end{array}$ & Erdnuss, mindestens Ara h 2 \\
\hline & Meeresfrüchten & Tropomyosin & Meeresfrucht, Pen a 1 \\
\hline \multirow{2}{*}{$\begin{array}{l}\text { Doppelpositivität Bie- } \\
\text { ne und Wespe }\end{array}$} & Biene & Api $m 1$ & \multirow{2}{*}{$\begin{array}{l}\text { Biene/Wespe, falls positiv: } \\
\text { 2. Api } \mathrm{m} 1 / \text { Ves } v 5 \\
\text { CCD }\end{array}$} \\
\hline & Wespe & Ves $v 5$ & \\
\hline Latexallergie & Latex & Hevein & Latex; mindestens Hev b 5/b 6 \\
\hline \multicolumn{4}{|c|}{$\begin{array}{l}\text { Die vorgeschlagenen Bestimmungen erheben keinen Anspruch aufVollständigkeit/Notwendigkeit und sind } \\
\text { abhängig von der Klinik und Gesamtsituation zu modifizieren. Weder kann durch diese Bestimmungen eine } \\
\text { heikle IgE-vermittelte Sensibilisierung völlig ausgeschlossen werden, noch sind umgekehrt schwere Reaktionen } \\
\text { zwingend. }\end{array}$} \\
\hline
\end{tabular}

Jahren für viele Allergene noch nicht die Sensitivität und hohe Reproduzierbarkeit einer herkömmlichen IgE-Bestimmung mittels Immuno-CAP gegenüber einem einzelnen Allergen erreicht wird.

Die Kosten für eine Chipanalyse liegen aktuell im Bereich von mehreren EinzelIgE-Bestimmungen, die wiederum von Land zu Land variieren. In Deutschland sind verschiedene Modelle mit Abrechnung nach IGel-Liste respektive für Privatpatienten möglich. Dabei kann das sog. Baukastenprinzip angewendet werden, da es sich um eine innovative, kostenintensive und mit einer komplexen Befundleistung des Arztes einhergehende Leistung handelt. Die Kosten können somit für den einzelnen Fall aktuell noch recht hoch werden und sollten mit dem Patienten sinnvollerweise vorher besprochen werden. Allerdings relativieren sich diese tel- und Latexallergien können Sensibilisierungen auf Proteine mit hohem Anaphylaxierisiko wie Speicher- oder Lipidtransferproteine identifiziert werden. Bei Hymenopterengiftallergien lassen sich Doppelsensibilisierungen auf Bienen- und Wespengift besser von Kreuzreaktivitäten abgrenzen (Übersicht in - Tab. 2).

Der Einsatz von gewissen rekombinanten Allergenen auch in der Routinediagnostik ist durchaus sinnvoll. Eine sorgfältige wissenschaftliche Untersuchung von deren Potenzial und Limitationen ist aber gerade deswegen umso notwendiger.

\section{Korrespondenzadresse}

\section{PD Dr. P. Schmid-Grendelmeier}

Dermatologische Klinik, Universitätsspital

Gloriastr. 31, 8091 Zürich

Schweiz

peter.schmid@usz.ch

Interessenkonflikt. Der korrespondierende Autor weist auf folgende Beziehungen hin: Der Autor PD Dr. Peter Schmid-Grendelmeier wirkt als Berater für die Firmen Bühlmann AG, Siemens Diagnostics AG und Phadia AG und erhielt von diesen Firmen Honorare für Vorträge.

\section{Literatur}

1. Barber D, Torre F de la, Feo F et al (2008) Understanding patient sensitization profiles in complex pollen areas: a molecular epidemiological study. Allergy 63(11):1550-1558

2. Benhamou $\mathrm{AH}$, Caubet JC, Eigenmann PA et al (2010) State of the art and new horizons in the diagnosis and management of egg allergy. Allergy 65(3):283-289

3. Brans R, Ott H, Merk HF (2009) Weizenabhängige anstrengungsinduzierte Anaphylaxie. Hautarzt 60(12):956-960

4. Crameri R, Hemmann S, Ismail C et al (1998) Disease-specific recombinant allergens for the diagnosis of allergic bronchopulmonary aspergillosis. Int Immunol 10(8):1211-1216

5. Ebo DG, Hagendorens MM, De Knop KJ et al (2010) Component-resolved diagnosis from latex allergy by microarray. Clin Exp Allergy 40(2):348-358

6. Focke M, Marth K, Flicker S, Valenta R (2008) Heterogeneity of commercial timothy grass pollen extracts. Clin Exp Allergy 38(8):1400-1408

7. Focke M, Marth K, Valenta R (2009) Molecular composition and biological activity of commercial birch pollen allergen extracts. Eur J Clin Invest 39(5):429-436

8. Heiss S, Mahler V, Steiner R et al (1999) Component-resolved diagnosis (CRD) of type I allergy with recombinant grass and tree pollen allergens by skin testing. J Invest Dermatol 113(5):830-837

9. Hiller R, Laffer S, Harwanegg C et al (2002) Microarrayed allergen molecules: diagnostic gatekeepers for allergy treatment. FASEB J 16(3):414-416 
10. Kraft D, Ferreira F, Ebner C et al (1998) Recombinant allergens: the future of the diagnosis and treatment of atopic allergy. Allergy 53:62-66

11. Léonard R, Wopfner N, Pabst M et al (2010) A new allergen from ragweed (Ambrosia artemisiifolia) with homology to art v 1 from mugwort. J Biol Chem 285:27192-27200

12. Matsuo H, Dahlström J, Tanaka A et al (2008) Sensitivity and specificity of recombinant omega- $5 \mathrm{gli}$ adin-specific lgE measurement for the diagnosis of wheat-dependent exercise-induced anaphylaxis. Allergy 63(2):233-236

13. Menz G, Willer G, Crameri R (2000) Allergische bronchopulmonale Aspergillose (ABPA). Pneumologie 54(9):375-384

14. Müller UR, Johansen N, Petersen AB et al (2009) Hymenoptera venom allergy: analysis of double positivity to honey bee and vespula venom by estimation of IgE antibodies to species-specific major allergens Api $\mathrm{m} 1$ and Ves v5. Allergy 64(4):543-548

15. Ott H, Baron JM, Heise R et al (2008) Clinical usefulness of microarray-based lgE detection in children with suspected food allergy. Allergy 63(11):15211528

16. Ott H, Fölster-Holst R, Merk HF, Baron JM (2009) Allergen microarrays: a novel tool for high-resolution lgE profiling in adults with atopic dermatitis. Eur J Dermatol 20(1):54-61

17. Ott H, Schröder C, Raulf-Heimsoth M et al (2010) Microarrays of recombinant Hevea brasiliensis proteins: a novel tool for the component-resolved diagnosis of natural rubber latex allergy. J Investig Allergol Clin Immunol 20(2):129-138

18. Reese I, Zuberbier T, Bunselmeyer B et al (2009) Diagnostic approach for suspected pseudoallergic reaction to food ingredients. J Dtsch Dermatol Ges 7(1):70-77

19. Schmid-Grendelmeier P, Crameri R (2001) Recombinant allergens for skin testing. Int Arch Allergy Immunol 125(2):96-111

20. Schmid-Grendelmeier P, Holzmann D, Himly M et al (2003) Native Art v 1 and recombinant Art v 1 are able to induce humoral and T cell-mediated in vitro and in vivo responses in mugwort allergy. J Allergy Clin Immunol 111(6):1328-1336

21. Valenta R, Laffer S, Vrtala S et al (1996) Recombinant allergens. Steps on the way to diagnosis and therapy of type I allergy. Adv Exp Med Biol 409:185-196

22. Wopfner N, Gadermaier G, Egger M et al (2005) The spectrum of allergens in ragweed and mugwort pollen. Int Arch Allergy Immunol 138:337346

\section{Möchten Sie einen Beitrag für „Der Hautarzt" einreichen?}

Wir freuen uns, dass Sie unsere Zeitschrift „Der Hautarzt“ mitgestalten möchten. Um Ihnen bei der Manuskripterstellung behilflich zu sein, haben wir für unsere Autoren Hinweise zusammengestellt, die Sie im Internet finden unter www.DerHautarzt.de (Für Autoren). Bitte senden Sie Ihren fertigen Beitrag an:

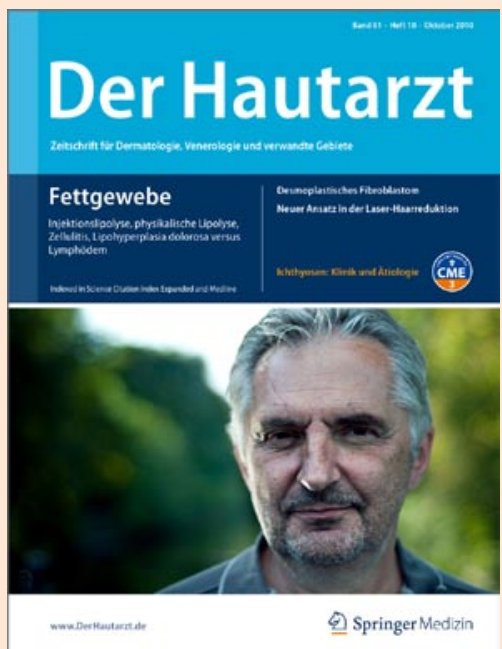

Originalien:

Prof. Dr. Alexander Kapp

Klinik für Dermatologie, Allergologie und Venerologie, Medizinische Hochschule Hannover, Ricklinger Straße 5, 30449 Hannover

Tel: +49-511-9246-232

Fax: +49-511-9246-234

freimooser.martina@mh-hannover.de

Übersichten, Kasuistiken:

Prof. Dr. Thomas Ruzicka

Klinik und Poliklinik für Dermatologie, LMU München

Anfragen an:

Prof. Dr. Daniela Bruch-Gerharz

Hautklinik des Universitätsklinikums

Düsseldorf, Moorenstr. 5,

40225 Düsseldorf

Tel: +49-211-81-18328

Fax: +49-211-81-04905

s.gehrke@med.uni-duesseldorf.de
Weiterbildung.

Zertifizierte Fortbildung:

Anfragen an:

Prof. Dr. Michael Meurer

Klinik und Poliklinik für Dermatologie an der Universitätsklinik Carl Gustav

Carus,

TU Dresden, Fetscherstr. 74, 01307 Dresden

meurer@rcs.urz.tu-dresden.de

Prof. Dr. Sonja Ständer

Klinik und Poliklinik für Hautkrankheiten, Universitätsklinikum Münster von-Esmarch-Str. 58 48149 Münster sonja.staender@uni-muenster.de

Prof. Dr. Rolf-Markus Szeimies Klinik für Haut-, Allergie-, Venen- und Umwelterkrankungen,

Knappschaftskrankenhaus

Recklinghausen, Klinikum Vest GmbH, Dorstener Str. 151, 45657 Recklinghausen dermatologie@kk-recklinghausen.de

Wie lautet Ihre Diagnose?:

Prof. Dr. Karin Scharffetter-Kochanek Universitätsklinik und Poliklinik für Dermatologie, Abteilung für Dermatologie und Allergologie, Maienweg 12, $89081 \mathrm{UIm}$

Tel: +49-731-500-21801

Fax: +49-731-500-21870

In der Diskussion:

Prof. Dr. Alexander Kapp/

Prof. Dr. Thomas Werfel

Klinik für Dermatologie, Allergologie und Venerologie,

Medizinische Hochschule Hannover,

Ricklinger Straße 5, 30449 Hannover freimooser.martina@mh-hannover.de

Leserforum:

Prof. Dr. Hans F. Merk

Klinik für Dermatologie und

Allergologie, Universitätsklinikum der RWTH, Pauwelsstraße 30, 52057 Aachen Hans.Merk@post.rwth-aachen.de

Gesundheitsökonomie:

Prof. Dr. Michael Jünger

Klinik und Poliklinik für Hautkrankheiten, Klinikum der Ernst-MoritzArndt-Universität Greifswald, Fleischmannstraße 42-44, 17487 Greifswald juenger@uni-greifswald.de 